\title{
Quantification of finite-temperature effects on adsorption geometries of $\pi$-conjugated molecules: Azobenzene/Ag(111)
}

\author{
G. Mercurio, ${ }^{1,2, *}$ R. J. Maurer, ${ }^{3}$ W. Liu ${ }^{4}$ S. Hagen, ${ }^{5}$ F. Leyssner, ${ }^{5}$ P. Tegeder, ${ }^{5,6}$ J. Meyer, ${ }^{3}$ \\ A. Tkatchenko, ${ }^{4}$ S. Soubatch, ${ }^{1,2}$ K. Reuter, ${ }^{3}$ and F. S. Tautz ${ }^{1,2}$ \\ ${ }^{1}$ Peter Grünberg Institut (PGI-3), Forschungszentrum Jülich, 52425 Jülich, Germany \\ ${ }^{2}$ Jülich Aachen Research Alliance (JARA), Fundamentals of Future Information Technology, 52425 Jülich, Germany \\ ${ }^{3}$ Department Chemie, Technische Universität München, Lichtenbergstraße 4, 85747 Garching, Germany \\ ${ }^{4}$ Fritz-Haber-Institut der Max-Planck-Gesellschaft, Faradayweg 4-6, 14195 Berlin, Germany \\ ${ }^{5}$ Freie Universität Berlin, Fachbereich Physik, Arnimallee 14, 14195 Berlin, Germany \\ ${ }^{6}$ Physikalisch-Chemisches Institut, Ruprecht-Karls-Universität Heidelberg, Im Neuenheimer Feld 253, 69120 Heidelberg, Germany
}

(Received 7 March 2013; published 12 July 2013)

\begin{abstract}
The adsorption structure of the molecular switch azobenzene on $\operatorname{Ag}(111)$ is investigated by a combination of normal incidence $\mathrm{x}$-ray standing waves and dispersion-corrected density functional theory. The inclusion of nonlocal collective substrate response (screening) in the dispersion correction improves the description of dense monolayers of azobenzene, which exhibit a substantial torsion of the molecule. Nevertheless, for a quantitative agreement with experiment explicit consideration of the effect of vibrational mode anharmonicity on the adsorption geometry is crucial.
\end{abstract}

DOI: 10.1103/PhysRevB.88.035421

PACS number(s): 68.43.Fg, 68.43.Pq, 68.49.Uv, 71.15.Mb

Precise experimentally determined structures of large organic adsorbates are indispensable for the detailed understanding of their wide-ranged functionalities, but also for benchmarking $a b$ initio electronic structure calculations. ${ }^{1-3}$ For large molecules with polarizable $\pi$-electron systems, van der Waals (vdW) interactions are substantial and may critically influence the adsorption geometry. ${ }^{4-7}$ Accounting for these interactions in ab initio calculations remains a challenge, and different approaches to this problem at varying degrees of accuracy are currently explored. ${ }^{8-14}$ Due to the system sizes inherent to large molecular adsorbates, efficient semiempirical dispersion correction schemes to density functional theory (SEDC-DFT) are particularly promising. ${ }^{13}$ However, their approximate nature makes them even more dependent on reliable experimental benchmark structures. This holds in particular for adsorption at metal surfaces, where the nonlocal collective substrate response (many-body electronic screening) requires advancements beyond the traditional pairwise summation of vdW interactions in these schemes. ${ }^{14,15}$

With SEDC-DFT now striving for the approximate inclusion of the collective substrate response, ${ }^{14}$ the accuracy increases to approximately $0.1 \AA$ for the predicted adsorption heights. ${ }^{14,16-18}$ At this level of accuracy, a new issue arises: Experiments for structure determination are often carried out close to room temperature, while in SEDC-DFT the ground state $($ at $0 \mathrm{~K})$ is normally calculated. The complex internal vibrational structure of large organic adsorbates which may sensitively influence the experimental time-averaged geometry is thus neglected. In this paper we show that the inclusion of such thermal expansion effects into SEDC-DFT is indeed necessary to reach quantitative agreement between experiment and theory. Hence, benchmarking at the current level of sophistication requires the careful analysis of finitetemperature effects. Otherwise misleading conclusions with respect to the SEDC-DFT accuracy might be obtained.

Our experiments have been carried out on azobenzene [AB; see Fig. 1(a)] adsorbed at $\operatorname{Ag}(111)$, by the normal incidence $\mathrm{x}$-ray standing wave technique (NIXSW). AB is a widely used molecular switch. ${ }^{20}$ Investigations of its substrate interaction are driven by the challenge to preserve the switching functionality in the presence of a surface. In this context, the knowledge of the adsorption structure is essential. NIXSW is an established method to determine the adsorption geometry (in particular adsorption heights) of large organic adsorbates. ${ }^{21,22}$ The $\mathrm{AB} / \mathrm{Ag}(111)$ system has been studied by NIXSW before and the results were compared to the SEDC-DFT approaches of the time to conclude on the importance of (then untreated) electronic screening effects. ${ }^{15}$ Using a most recent SEDC-DFT revision that approximately includes nonlocal collective substrate response we here confirm this proposition. Also, accounting in our refined analysis for coverage dependence, we nevertheless show that the crucial missing link to achieve quantitative agreement with experiment lies not on the electronic structure level, but in hitherto generally neglected finite-temperature effects.

NIXSW experiments were carried out at ESRF, beamline ID32, under ultrahigh-vacuum conditions $\left(\approx 5 \times 10^{-10} \mathrm{mbar}\right) .{ }^{15}$ The $\mathrm{Ag}(111)$ surface was cleaned by several cycles of $\mathrm{Ar}^{+}$ion sputtering and annealing at $820 \mathrm{~K}$. Multilayers of $\mathrm{AB}$ were deposited from an effusion cell held at room temperature onto the atomically ordered $\mathrm{Ag}(111)$ crystal at $220 \mathrm{~K}$. AB monolayers were then prepared by desorption from multilayers, by heating with a rate of $1 \mathrm{~K} / \mathrm{s}$ until the multilayer desorption peak had decayed and before the monolayer peak was observed. ${ }^{23}$ This guarantees coverages close to one monolayer. The $\mathrm{AB}$ fragment mass of $77 \mathrm{amu}\left(\mathrm{C}_{6} \mathrm{H}_{5}^{+}\right)$was monitored online with a quadrupole mass spectrometer. The Ag crystal was kept at $210 \mathrm{~K}$ during the NIXSW experiments to prevent desorption.

Vibrations are expected to influence the average geometry of the adsorbate (via vibrational mode anharmonicity) and to broaden the distribution of atoms around their average positions (via vibrational dynamics). ${ }^{21}$ While this will affect both the coherent position $P_{c}$ and the coherent fraction $F_{c}$ of 

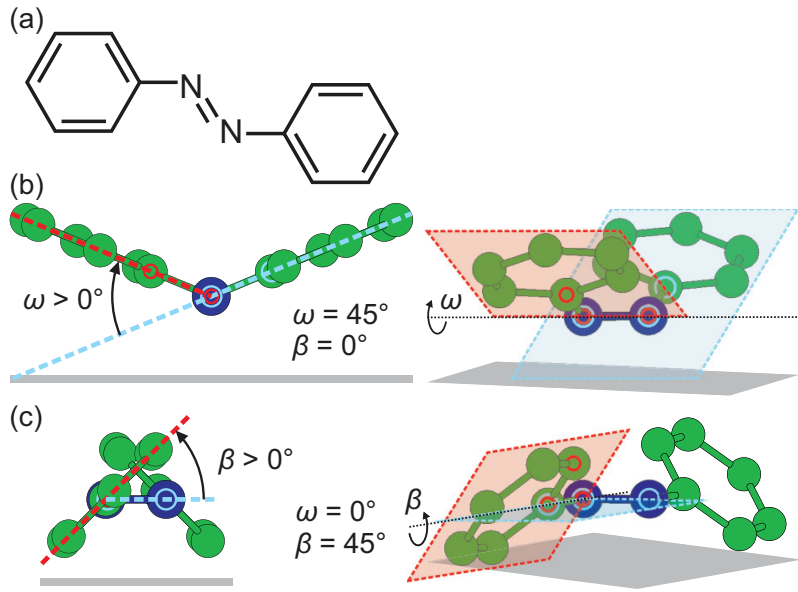

FIG. 1. (Color) (a) Structure formula of azobenzene (AB). (b) Side view and perspective view of $\mathrm{AB}$ with $\omega=45^{\circ}$ and $\beta=0^{\circ}$. (c) Side view and perspective view of $\mathrm{AB}$ with $\omega=0^{\circ}$ and $\beta=45^{\circ} . \omega$ and $\beta$ are defined as the dihedral angles CNNC and CCNN (Ref. 19), respectively. The planes containing atoms $\mathrm{C}, \mathrm{N}, \mathrm{N}$ (b) and $\mathrm{C}, \mathrm{C}, \mathrm{N}$ (c) are marked in red and the corresponding atoms are indicated by red circles. The planes containing atoms N, N, C (b) and C, N, N (c) are marked in light blue and the corresponding atoms are indicated by light blue circles. $\mathrm{C}$ atoms: green spheres. $\mathrm{N}$ atoms: blue spheres. For clarity, $\mathrm{H}$ atoms are not drawn.

the NIXSW signal, prevalent (harmonic) Debye-Waller theory only considers temperature effects on $F_{c}{ }^{21,24} P_{c}$ defines the average adsorption height of a species, while $F_{c}$ quantifies the corresponding height distribution. A coherent fraction of 1 means that all photoemitters of a certain species have precisely the same adsorption height above the relevant family of Bragg planes, while a coherent fraction of 0 indicates a homogeneous distribution of the photoemitters throughout the Bragg spacing. In general, $F_{c}<1$ due to unavoidable structural disorder ${ }^{25}$ and adsorbate and substrate thermal vibrations. ${ }^{26}$ However, the coherent fractions of different chemical species also contain information about the internal geometry of the adsorbate that has so far been left aside in most NIXSW studies. Here we recover this information by including differences between the $F_{c}$ of different species into our analysis.

In the present case of $\mathrm{AB} / \mathrm{Ag}(111)$, NIXSW provides coherent positions $P_{c}^{\mathrm{C}}=0.27 \pm 0.02, \quad P_{c}^{\mathrm{N}}=0.26 \pm 0.02$ and coherent fractions $F_{c}^{\mathrm{C}}=0.34 \pm 0.03, F_{c}^{\mathrm{N}}=0.48 \pm 0.12$ (Fig. 2). ${ }^{27}$ For the general procedure followed here in order to determine the structure parameters $\left(P_{c}, F_{c}\right)$ and their error bars, we refer to Ref. 25. The programs TORRICELLI ${ }^{23,28,29}$ and CasaXPS ${ }^{30}$ have been employed. The fitting models for nitrogen and carbon photoemission spectra are reported in Refs. 23 and 31. We note that calculated nondipolar parameters have been used ${ }^{26,32-35}$ instead of experimentally measured ones, because the latter may produce ambiguous results. ${ }^{36-38}$

While the respective coherent positions are identical within the errors, the coherent fraction of $\mathrm{C}$ is $29 \%$ smaller than the one of $\mathrm{N}$. In our refined structure determination, we ascribe this difference to the internal geometry of $\mathrm{AB}$, assuming that $F_{c}^{\mathrm{C}_{i}}$ and $F_{c}^{\mathrm{N}_{i}}$, the coherent fractions of individual $\mathrm{C}$ and $\mathrm{N}$ atoms, are equal (and smaller than 1 due to disorder). ${ }^{26}$ To solve the $\mathrm{AB}$ structure, two internal degrees of freedom are

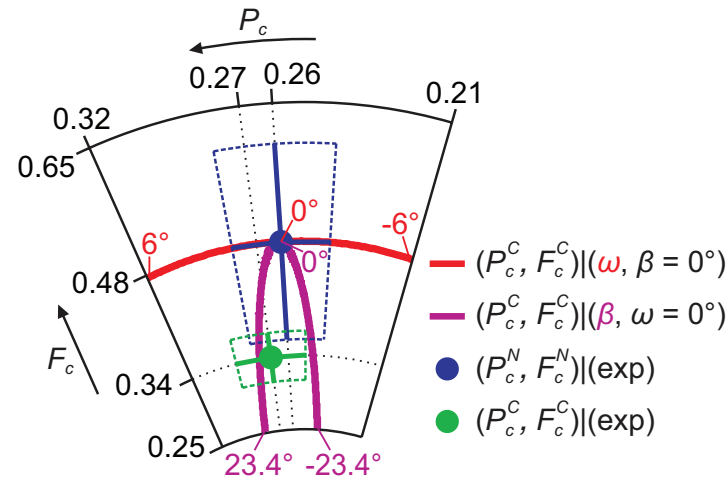

FIG. 2. (Color) Argand diagram indicating the NIXSW experimental results and NIXSW simulations for $\mathrm{AB} / \mathrm{Ag}(111)$. Green filled circle: Average experimental $\left(P_{c}, F_{c}\right)=(0.27 \pm 0.02,0.34 \pm 0.03)$ of carbon. Blue filled circle: Average experimental $\left(P_{c}, F_{c}\right)=(0.26 \pm$ $0.02,0.48 \pm 0.12)$ of nitrogen. Green and blue solid lines: Corresponding error bars. Green and blue dashed lines: Corresponding error regions (Ref. 26). Red solid line: Simulated $\left(P_{c}, F_{c}\right)$ of carbon with $-6^{\circ} \leqslant \omega \leqslant 6^{\circ}$ and $\beta=0^{\circ}$. Magenta solid line: Simulated $\left(P_{c}\right.$, $F_{c}$ ) of carbon with $-23.4^{\circ} \leqslant \beta \leqslant 23.4^{\circ}$ and $\omega=0^{\circ}$.

considered: the tilt angle $\omega$ [Fig. 1(a) $\left.{ }^{39}\right]$ and the torsion angle $\beta$ [Fig. 1(b)], defined as dihedral angles CNNC and CCNN, ${ }^{19}$ respectively. It is impossible to explain the ratio $F_{c}^{C} / F_{c}^{N}$ in a model in which $\omega$ is the only internal degree of freedom of the molecule, because any distortion along $\omega$ that would lead to a decrease of $F_{c}^{\mathrm{C}}$ would at the same time result in an increase of the coherent position $P_{c}^{\mathrm{C}}$ which is related to the average adsorption height of the carbon atoms. Hence, an additional degree of freedom must be considered to explain the measured NIXSW structure parameters. A plausible choice is the torsion angle $\beta$, because for small angles $\omega$ a finite $\beta$ would broaden the carbon distribution essentially without changing the average carbon height (Fig. 2, magenta curve). Note that this broadening could in principle be due to a static distortion of the molecule and/or due to its vibrational dynamics. However, for a purely dynamical reduction of the average coherent fraction $F_{c}^{\mathrm{C}}$ by $29 \%$ an unreasonably large $\mathrm{C}$ vibrational amplitude of the order $\pm 0.30 \AA$ (with fixed $\mathrm{N}$ atoms) would be required. Therefore, we will first consider a static distortion before coming back to a possible dynamical contribution.

Requiring $F_{c}^{\mathrm{C}_{i}}=F_{c}^{\mathrm{N}_{i}}$ and constructing the molecular geometry such that the measured values for $P_{c}^{\mathrm{C}}, P_{c}^{\mathrm{N}}, F_{c}^{\mathrm{C}}, F_{c}^{\mathrm{N}}$ are obtained, we find an adsorption geometry with $d_{\mathrm{N}-\mathrm{Ag}}$ of $2.97 \pm 0.05 \AA$, a tilt angle $\omega$ of $-0.7^{\circ}$, and a torsion angle $\beta$ of $17.7^{\circ}$ from our NIXSW data (see Table I). ${ }^{40,41}$ Note that in deriving these geometry parameters, we have assumed that both nitrogen atoms adsorb at the same height. ${ }^{42}$

A torsion angle $\beta$ of more than $17^{\circ}$ is difficult to rationalize for a single molecule adsorbed on the surface without neighbors. Yet, all calculations so far $^{15,43,44}$ have been carried out for single molecules (while our experiment is performed on a condensed layer; see above). We therefore need to analyze the coverage- and packing-dependence of the adsorption geometry of $\mathrm{AB} / \mathrm{Ag}(111)$ theoretically. While our previous SEDC-DFT calculations ${ }^{15}$ for this system were carried out at the level of the dispersion-correction scheme by Tkatchenko and Scheffler 
TABLE I. Summary of the geometry parameters $d_{\mathrm{N}-\mathrm{Ag}}, \omega$, and $\beta$ calculated by the two different SEDC-DFT schemes PBE + TS and $\mathrm{PBE}+\mathrm{vdW}$ surf for phase $\mathrm{LC}$ at $0 \mathrm{~K}$, and phase A at $0 \mathrm{~K}$ and $210 \mathrm{~K}$. Also shown are the experimental NIXSW results. $d_{\mathrm{N}-\mathrm{Ag}}$ are calculated as $\left(1+P_{c}\right) \times d_{\mathrm{Ag}(111)}$, with $d_{\mathrm{Ag}(111)}=2.3552 \AA$ (Refs. 26,57,58). Details concerning the calculation of $\omega, \beta$ and of all experimental error bars are reported in the Supplemental Material (Ref. 26).

\begin{tabular}{lcccc}
\hline \hline & & $d_{\mathrm{N}-\mathrm{Ag}} \AA$ & $\omega\left(^{\circ}\right)$ & $\beta\left(^{\circ}\right)$ \\
\hline phase LC & $\mathrm{TS}$ & 2.95 & 1.8 & -0.6 \\
$(T=0 \mathrm{~K})$ & $\mathrm{vdW}^{\text {surf }}$ & 2.61 & 4.5 & -2.0 \\
phase A & $\mathrm{TS}$ & 3.26 & 7.5 & 18.6 \\
$(T=0 \mathrm{~K})$ & $\mathrm{vdW}^{\text {surf }}$ & 2.81 & 11.7 & 15.4 \\
phase A & $\mathrm{TS}$ & 3.23 & 8.8 & 17.3 \\
$(T=210 \mathrm{~K})$ & $\mathrm{vdW}^{\text {surf }}$ & 2.98 & 9.0 & 17.7 \\
experiment & $\mathrm{NIXSW}$ & 2.97 & -0.7 & 17.7 \\
$(T=210 \mathrm{~K})$ & & \pm 0.05 & $(+2.3 /-2.2)$ & $(+2.4 /-2.7)$ \\
\hline \hline
\end{tabular}

(TS), ${ }^{12}$ we now employ the more recent vdW ${ }^{\text {surf }}$ scheme, ${ }^{14}$ which accounts for nonlocal collective substrate response via renormalization of the dispersion coefficients on the basis of Lifshitz-Zaremba-Kohn theory. Details of the calculations can be found in the Supplemental Material. ${ }^{26,45-52}$ We determine the optimized adsorption geometries for a range of different surface unit cells, ${ }^{26}$ with one AB per $(6 \times 7)$ cell representing the low-coverage $(\mathrm{LC})$ limit and two AB molecules in a $(2 \times 5)$ cell leading to the highest considered molecular surface density (see Fig. 3).

(b)

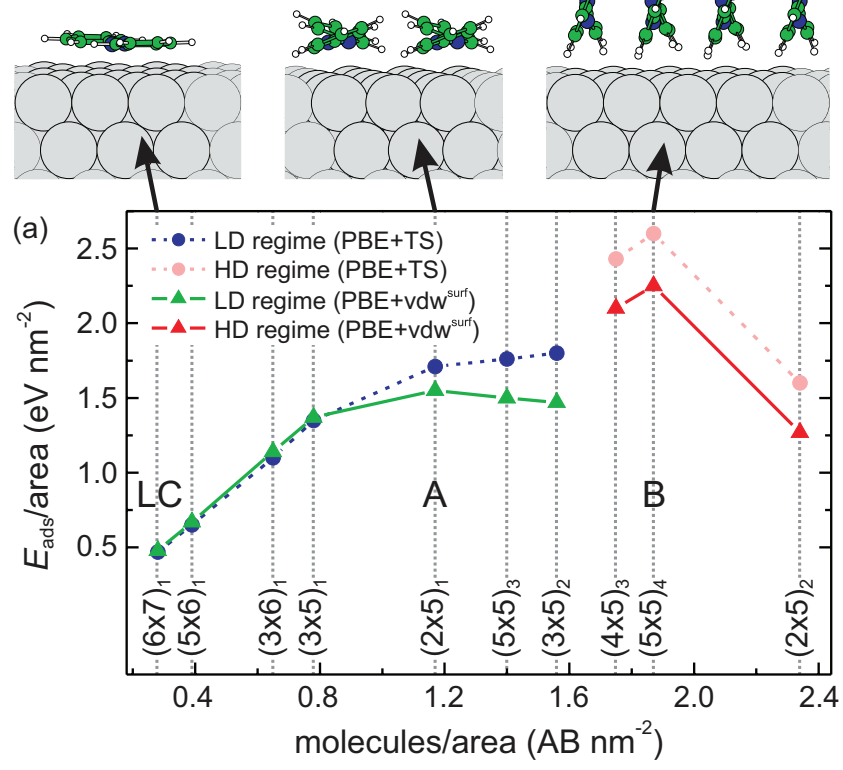

FIG. 3. (Color) (a) Adsorption energy per surface area vs surface coverage of $\mathrm{AB} / \mathrm{Ag}(111)$ as calculated with $\mathrm{PBE}+\mathrm{vdW}^{\text {surf }}$ (filled triangles, solid line) and PBE + TS (filled circles, dashed line). The low-density (LD) and high-density (HD) coverage regimes (see text) are indicated by different colors (green/blue and red/pink). Corresponding unit cells and the numbers $n$ of $\mathrm{AB}$ molecules in the unit cell are given as $(X \times Y)_{n}$. Adsorption geometry ( $\left.\mathrm{vdW}^{\text {surf }}\right)$ of the low-coverage (LC) phase (b), of phase A (c), and of phase $B(d)$.
The PBE $+v^{\text {dW }}{ }^{\text {surf }}$ results compiled in Fig. 3 show that the adsorption geometry indeed varies substantially with increasing molecular surface density. While in the LC limit the adsorbed molecule is essentially flat [Fig. 3(b)], the tilt and torsion angles $\omega$ and $\beta$ increase with the packing density [Figs. 3(c) and 3(d)]. As a consequence of the internal distortion of the molecule, the vertical adsorption height of the azo-bridge also increases; ${ }^{26}$ this tendency continues beyond the critical coverage of $1.56 \mathrm{AB} \cdot \mathrm{nm}^{-2}$ at which the now nearly upright molecules start to flatten out again within the increasingly dense overlayer [Fig. 3(d)]. The flattening of the upright molecule implies an increasingly asymmetric position of the azo-bridge, i.e., different vertical adsorption heights of the two nitrogen atoms, with a consequent lifting from the surface. ${ }^{26}$

With most of the adsorption energy of the flat $A B$ molecule in the LC limit coming from dispersive interactions with the substrate, the binding energy per molecule naturally decreases in the distorted high-density structures (see Supplemental Material, Table $\mathrm{I}^{26}$ ). Due to the denser packing, the adsorption energy per surface area $E_{\text {ads }} /$ area nevertheless increases and reaches a maximum at $1.17 \mathrm{AB} \cdot \mathrm{nm}^{-2}$ [Fig. 3(a)]. The intermolecular vdW interactions in the high-density phases further increase $E_{\text {ads }} /$ area, which reaches a second maximum at a density of $1.87 \mathrm{AB} \cdot \mathrm{nm}^{-2}$. Our calculations therefore predict the existence of two optimum packing densities, a phase A [Fig. 3(c)] at $1.17 \mathrm{AB} \cdot \mathrm{nm}^{-2}$ and a phase B [Fig. 3(d)] at $1.87 \mathrm{AB} \cdot \mathrm{nm}^{-2}$. Qualitatively similar findings are obtained with the TS scheme. In detail, however, there are decisive differences. For example, TS fails to predict the maximum of $E_{\text {ads }} /$ area corresponding to phase A; see Fig. 3(a).

To decide which structure-if any of the above-we have in our experiment, we take the calculated groundstate geometries and compare them to experiment (Table I). Phase LC can be ruled out, both because of its small torsion angle and because of our sample preparation procedure which yields dense layers. The average adsorption height of $\mathrm{N}$ atoms in phase $\mathrm{B}$ is $4.39 \AA$, which is inconsistent with the experimental value of $2.97 \AA$ or-modulo a Bragg spacing$5.32 \AA$. We therefore conclude that our NIXSW experiment has been carried out on a structure similar to phase A. This conclusion is consistent with the expectation that neither of the two dispersion-correction schemes will work reliably at the packing density of phase $B$ that is close to the one of the molecular crystal, in which even the vdW ${ }^{\text {surf }}$ scheme will overestimate lateral interactions, ${ }^{53}$ because higher order many-body terms are neglected. ${ }^{54}$ This neglect will contribute to a spurious stabilization of phase $\mathrm{B}$ in the calculation.

In Table I the geometry parameters of phase $\mathrm{A}$ are summarized. At $0 \mathrm{~K}$ the $\mathrm{vdW}^{\text {surf }}$ scheme yields a height of $d_{\mathrm{N}-\mathrm{Ag}}=2.81 \AA$ at tilt $\omega=11.7^{\circ}$ and torsion $\beta=15.4^{\circ}$, while the TS scheme predicts $d_{\mathrm{N}-\mathrm{Ag}}=3.26 \AA, \omega=7.5^{\circ}$, and $\beta=18.6^{\circ}$. With regard to $\beta$, we observe a good agreement of the ground-state calculation with the experimental result $\left(\beta=17.7^{\circ}\right)$. In contrast, the calculated adsorption heights of the azo-bridge are $0.16 \AA$ too small for vdW ${ }^{\text {surf }}$ and $0.29 \AA$ too large for TS. It is clear that the inclusion of collective substrate response has a large impact on the predicted adsorption height $d_{\mathrm{N}-\mathrm{Ag}}$. It tends to improve the TS prediction, although the height is still not perfect, and the calculated $\omega$ is too large. 
We will now show that the predictions of the vdW $\mathrm{Wurf}^{\text {theory }}$ can be substantially improved toward a quantitative agreement with experiment, if the effect of finite temperature is taken into account. In particular, anharmonic contributions to the vibrational motion may modify the time-averaged geometries that are experimentally observable. ${ }^{21}$ We demonstrate this by explicitly calculating the harmonic vibrations for the adsorbed $\mathrm{AB}$ molecule at the optimum density of $1.17 \mathrm{AB} \cdot \mathrm{nm}^{-2}$, both at $\mathrm{PBE}+\mathrm{vdW}$ surf and $\mathrm{PBE}+\mathrm{TS}$ levels. We then map out the anharmonic regimes of these modes at energies around the experimentally employed $210 \mathrm{~K}$, by distorting the molecule along the corresponding vibrational eigenvectors. Next, we fit a Morse potential ${ }^{55}$ to these data points for every harmonic mode and integrate the motion in the Morse potentials analytically to obtain the shifts of the average positions at $210 \mathrm{~K}$ relative to the harmonic minima. Summing these shifts over all vibrational modes, we finally construct an anharmonically corrected geometry for the adsorbed AB molecule. ${ }^{26}$ Note that the vibrational dynamics of the substrate is taken into account in the fitting of the photoelectron yield profiles of the NIXSW experiment at the level of Debye-Waller theory, ${ }^{24}$ with parameters from Ref. 56.

With this procedure we arrive at the following finitetemperature $(210 \mathrm{~K})$ structures for the $\mathrm{vdW}^{\text {surf }}$ (TS) schemes: $\quad d_{\mathrm{N}-\mathrm{Ag}}=2.98 \AA(3.23 \AA), \omega=9.0^{\circ}\left(8.8^{\circ}\right)$, and $\beta=17.7^{\circ}\left(17.3^{\circ}\right)$ (Table I). Driven particularly by the lowenergy adsorbate-substrate stretching modes, anharmonic effects primarily affect $d_{\mathrm{N}-\mathrm{Ag}}$. In the case of $\mathrm{vdW}$ surf , they lift the azo-bridge by $0.17 \AA$ into almost perfect agreement with the measured value of $2.97 \pm 0.05 \AA$. At the same time, the larger vertical adsorption height of the azo-bridge allows the molecule to flatten out again under the influence of the van der Waals interaction with the metal (reduction of $\omega$ ), and to twist further as a result of intermolecular interactions (increase of $\beta$ ). Both tendencies bring the calculated geometry closer to experiment, although the calculated $\omega$ remains too large. For $\mathrm{TS}$, on the other hand, anharmonicity affects $d_{\mathrm{N}-\mathrm{Ag}}$ and $\beta$ only mildly, because $d_{\mathrm{N}-\mathrm{Ag}}$ is too large even in the $0 \mathrm{~K}$ calculation; moreover, it has an adverse effect on $\omega$, because it brings the molecule closer to the surface. Overall, the quality gap between $\mathrm{vdW}^{\text {surf }}$ and TS is therefore widened by the inclusion of anharmonic effects.

To check the self-consistency of the finite-temperature geometry, we have simulated NIXSW results on its basis, with the aim to evaluate the influence of vibrational excitations on the coherence of the NIXSW signal (see Supplemental Material, Sec. III $^{26}$ for details). Note that our experimental values for $\omega$ and $\beta$ in Table I are based on the assumption that $F_{c}^{\mathrm{C}}$ and $F_{c}^{\mathrm{N}}$ are different exclusively due to static distortions. For the anharmonically corrected average structure of the molecule, the NIXSW simulation yields a $F_{c}^{\mathrm{C}} / F_{c}^{\mathrm{N}}=0.60(0.61)$ (TS in parentheses), a value very close to both experiment $(0.71)$ and the $0 \mathrm{~K}$ structure $\left(0.61\right.$ in vdW $\left.\mathrm{vWr}^{\text {surf }}\right)$. Most importantly, the reduction of the coherent fractions due to vibrational motion around the average structure is similar for $\mathrm{C}$ and $\mathrm{N}$, and approximately equal to $10 \%(5 \%)$ (TS in parentheses), such that $F_{c}^{\mathrm{C}} / F_{c}^{\mathrm{N}}$ becomes $0.63(0.62)$ (see Supplemental Material, Table IV $^{26}$ ), hence closer to experiment. The nearly equal reduction of $F_{c}^{\mathrm{C}}$ and $F_{c}^{\mathrm{N}}$ due to thermal vibrations confirms $a$ posteriori that in deriving the experimental structure we can interpret the different experimental $F_{c} \mathrm{~s}$ as being due to static distortion, and hence the experimental geometry parameters in Table I are the correct reference point for the calculated finite-temperature geometry.

In conclusion, we have analyzed the structure of the archetypal molecular switch azobenzene on the $\operatorname{Ag}(111)$ surface. We find that the inclusion of collective substrate response into SEDC-DFT correction schemes is absolutely essential for a correct description of the adsorption geometry. However, since the $\mathrm{vdW}^{\text {surf }}$ scheme leads to a reduction of both the dispersion coefficients and the van der Waals radii, it may-counterintuitively-decrease the adsorption height compared to SEDC-DFT without collective substrate response. This is clearly observed for the LC phase. However, we have identified two effects which increase the adsorption height again. First, this is the dense molecular packing and the associated molecular distortion, which increase $d_{\mathrm{N}-\mathrm{Ag}}$ by $0.20 \AA$. Second, the anharmonicity of molecular vibrations raises $d_{\mathrm{N}-\mathrm{Ag}}$ by another $0.17 \AA$. The remaining disagreements between experiment and theory notwithstanding, there are three important findings which can be generalized: First, information regarding the molecular conformation beyond the average positions of certain chemical species can be retrieved from the coherence of the NIXSW signal with suitable simulations. Second, thermal expansion due to the anharmonicity of molecular vibrations not captured in DebyeWaller theory ${ }^{21}$ may contribute $0.1-0.2 \AA$ to the adsorption height. This must be taken into account in future benchmarks of high-level $a b$ initio theory against NIXSW, either by carrying out the experiments at low temperature or by inclusion of finite-temperature vibrational effects into the calculation, as sketched in the present paper. And third, our observation that all three geometry parameters $d_{\mathrm{N}-\mathrm{Ag}}, \omega$, and $\beta$ develop into the correct direction if anharmonic effects are included proves that the $\mathrm{PBE}+\mathrm{vdW}$ surf scheme captures the essential physics of both chemical and dispersion interactions and is therefore a good starting point as a ground-state calculation for the adsorption of large $\pi$-conjugated molecules.

We acknowledge financial support of the Deutsche Forschungsgemeinschft TA244/3-2, SFB 658, and RE1509/16-1.
*Current address: University of Hamburg and Center for FreeElectron Laser Science, Luruper Chausse 149, 22761 Hamburg, Germany; giuseppe.mercurio@desy.de

${ }^{1}$ F. S. Tautz, Prog. Surf. Sci. 82, 479 (2007).

${ }^{2}$ L. Romaner, G. Heimel, J.-L. Brédas, A. Gerlach, F. Schreiber, R. L. Johnson, J. Zegenhagen, S. Duhm, N. Koch, and E. Zojer, Phys. Rev. Lett. 99, 256801 (2007).
${ }^{3}$ N. Koch, A. Gerlach, S. Duhm, H. Glowatzki, G. Heimel, A. Vollmer, Y. Sakamoto, T. Suzuki, J. Zegenhagen, J. P. Rabe, and F. Schreiber, J. Am. Chem. Soc. 130, 7300 (2008).

${ }^{4}$ N. Atodiresei, V. Caciuc, P. Lazić, and S. Blügel, Phys. Rev. Lett. 102, 136809 (2009).

${ }^{5}$ F. Mittendorfer, A. Garhofer, J. Redinger, J. Klimeš, J. Harl, and G. Kresse, Phys. Rev. B 84, 201401 (2011). 
${ }^{6}$ W. Liu, A. Savara, X. Ren, W. Ludwig, K.-H. Dostert, S. Schauermann, A. Tkatchenko, H.-J. Freund, and M. Scheffler, J. Phys. Chem. Lett. 3, 582 (2012).

${ }^{7}$ P. Sony, P. Puschnig, D. Nabok, and C. Ambrosch-Draxl, Phys. Rev. Lett. 99, 176401 (2007).

${ }^{8}$ S. Grimme, J. Antony, S. Ehrlich, and H. Krieg, J. Chem. Phys. 132, 154104 (2010).

${ }^{9}$ K. Lee, E. D. Murray, L. Kong, B. I. Lundqvist, and D. C. Langreth, Phys. Rev. B 82, 081101 (2010).

${ }^{10}$ J. Klimeš, D. R. Bowler, and A. Michaelides, J. Phys.: Condens. Matter 22, 022201 (2010).

${ }^{11}$ S. N. Steinmann and C. Corminboeuf, J. Chem. Theory Comput. 7, 3567 (2011).

${ }^{12}$ A. Tkatchenko and M. Scheffler, Phys. Rev. Lett. 102, 073005 (2009).

${ }^{13}$ A. Tkatchenko, L. Romaner, O. T. Hofmann, E. Zojer, C. AmbroschDraxl, and M. Scheffler, MRS Bull. 35, 435 (2010).

${ }^{14}$ V. G. Ruiz, W. Liu, E. Zojer, M. Scheffler, and A. Tkatchenko, Phys. Rev. Lett. 108, 146103 (2012).

${ }^{15}$ G. Mercurio, E. R. McNellis, I. Martin, S. Hagen, F. Leyssner, S. Soubatch, J. Meyer, M. Wolf, P. Tegeder, F. S. Tautz, and K. Reuter, Phys. Rev. Lett. 104, 036102 (2010).

${ }^{16}$ W. Liu, J. Carrasco, B. Santra, A. Michaelides, M. Scheffler, and A. Tkatchenko, Phys. Rev. B 86, 245405 (2012).

${ }^{17}$ W. A. Al-Saidi, H. Feng, and K. A. Fichthorn, Nano Lett. 12, 997 (2012).

${ }^{18}$ C. Wagner, N. Fournier, F. S. Tautz, and R. Temirov, Phys. Rev. Lett. 109, 076102 (2012).

${ }^{19}$ In a chain of atoms A-B-C-D, the dihedral angle is the angle between the plane containing the atoms $\mathrm{A}, \mathrm{B}, \mathrm{C}$ and that containing $\mathrm{B}, \mathrm{C}$, $\mathrm{D}$ on a third plane normal to the intersection of the two planes (Ref. 59). In this paper, dihedral angles are defined modulo $180^{\circ}$.

${ }^{20}$ Molecular Switches, edited by B. L. Feringa and W. R. Browne, 2nd ed. (Wiley-VCH, Weinheim, 2011).

${ }^{21}$ J. Zegenhagen, Surf. Sci. Rep. 18, 202 (1993).

${ }^{22}$ D. P. Woodruff, Rep. Prog. Phys. 68, 743 (2005).

${ }^{23} \mathrm{G}$. Mercurio, Study of Molecule-Metal Interfaces by Means of the Normal Incidence X-Ray Standing Wave Technique, Ph.D. thesis, Schriften des Forschungszentrums Jülich, Reihe Schlüsseltechnologien Vol. 49 (Dissertation, RWTH Aachen University, 2012), available at http://www.fz-juelich.de/zb/juwel.

${ }^{24}$ D. P. Woodruff, B. C. C. Cowie, and A. R. H. F. Ettema, J. Phys.: Condens. Matter 6, 10633 (1994).

${ }^{25}$ G. Mercurio, O. Bauer, M. Willenbockel, N. Fairley, W. Reckien, C. H. Schmitz, B. Fiedler, S. Soubatch, T. Bredow, M. Sokolowski, and F. S. Tautz, Phys. Rev. B 87, 045421 (2013).

${ }^{26}$ See Supplemental Material at http://link.aps.org/supplemental/ 10.1103/PhysRevB.88.035421 for a detailed description of DFT calculations, NIXSW results, and simulations.

${ }^{27}$ The origin of the slight differences between NIXSW results published here and those based on the same data and published in Ref. 15 is discussed in the Supplemental Material (Ref. 26).

${ }^{28}$ TORRICELLI is an XSW data analysis and simulation program written by G. Mercurio; copies can be obtained from s.tautz@fz-juelich.de.

${ }^{29}$ G. Mercurio, N. Fairley, S. Soubatch, and F. S. Tautz (unpublished).

${ }^{30}$ N. Fairley, CasaXPS, version 2.3.16, Casa Software Ltd, Bay House, 5 Grosvenor Terrace, Teignmouth, Devon TQ14 8NE, UK.

${ }^{31}$ G. Mercurio, R. J. Maurer, S. Hagen, F. Leyssner, J. Meyer, M. Wolf, P. Tegeder, S. Soubatch, K. Reuter, and F. S. Tautz (unpublished).
${ }^{32}$ I. A. Vartanyants and J. Zegenhagen, Solid State Commun. 113, 299 (1999).

${ }^{33}$ J. J. Lee, C. J. Fisher, D. P. Woodruff, M. G. Roper, R. G. Jones, and B. C. C. Cowie, Surf. Sci. 494, 166 (2001).

${ }^{34}$ M. B. Trzhaskovskaya, V. I. Nefedov, and V. G. Yarzhemsky, At. Data Nucl. Data Tables 77, 97 (2001).

${ }^{35}$ A. Jablonski, F. Salvat, and C. J. Powell, NIST Electron ElasticScattering Cross-Section Database, Tech. Rep. (Version 3.1, National Institute of Standards and Technology, Gaithersburg, MD, 2003).

${ }^{36}$ A. Gerlach, F. Schreiber, S. Sellner, H. Dosch, I. A. Vartanyants, B. C. C. Cowie, T.-L. Lee, and J. Zegenhagen, Phys. Rev. B 71, 205425 (2005)

${ }^{37}$ C. Stadler, S. Hansen, F. Pollinger, C. Kumpf, E. Umbach, T.-L. Lee, and J. Zegenhagen, Phys. Rev. B 74, 035404 (2006).

${ }^{38}$ A. Gerlach, S. Sellner, F. Schreiber, N. Koch, and J. Zegenhagen, Phys. Rev. B 75, 045401 (2007).

${ }^{39}$ Note that in Ref. 15 we used a different tilt angle $\tilde{\omega}$ defined as the the angle between the $\mathrm{N}-\mathrm{C}$ bond of $\mathrm{AB}$ and the surface plane.

${ }^{40}$ Note that an alternative configuration with $\beta<0^{\circ}$ [see Fig. 1(b) of the Supplemental Material (Ref. 26)] is also consistent with experiment. It has a tilt angle $\omega$ of $2.6^{\circ}\left(+2.2^{\circ} /-2.3^{\circ}\right)$ and a torsion angle $\beta$ of $-18.0^{\circ}\left(+2.6^{\circ} /-2.4^{\circ}\right)$. SEDC-DFT predicts a positive $\beta$ for phase A.

${ }^{41}$ For the derivation of these geometry parameters from the measured NIXSW structure parameters, see Supplemental Material, Sec. II (Ref. 26).

${ }^{42}$ E. McNellis, J. Meyer, A. D. Baghi, and K. Reuter, Phys. Rev. B 80, 035414 (2009).

${ }^{43}$ G. Li, I. Tamblyn, V. R. Cooper, H.-J. Gao, and J. B. Neaton, Phys. Rev. B 85, 121409 (2012).

${ }^{44}$ E. R. McNellis, J. Meyer, and K. Reuter, Phys. Rev. B 80, 205414 (2009).

${ }^{45}$ H. J. Monkhorst and J. D. Pack, Phys. Rev. B 13, 5188 (1976).

${ }^{46}$ P. Hohenberg and W. Kohn, Phys. Rev. 136, B864 (1964).

${ }^{47}$ W. Kohn and L. J. Sham, Phys. Rev. 140, A1133 (1965).

${ }^{48}$ S. J. Clark, M. D. Segall, C. J. Pickard, P. J. Hasnip, M. I. J. Probert, M. C. Refson, and K. Payne, Z. Kristallogr. 220, 567 (2005).

${ }^{49}$ M. C. Payne, M. P. Teter, D. C. Allan, T. A. Arias, and J. D. Joannopoulos, Rev. Mod. Phys. 64, 1045 (1992).

${ }^{50}$ D. Vanderbilt, Phys. Rev. B 41, 7892 (1990).

${ }^{51}$ J. P. Perdew, K. Burke, and M. Ernzerhof, Phys. Rev. Lett. 77, 3865 (1996).

${ }^{52}$ J. Andzelm, R. King-Smith, and G. Fitzgerald, Chem. Phys. Lett. 335, 321 (2001).

${ }^{53}$ A. Tkatchenko and O. A. von Lilienfeld, Phys. Rev. B 78, 045116 (2008).

${ }^{54}$ A. Tkatchenko, R. A. DiStasio, R. Car, and M. Scheffler, Phys. Rev. Lett. 108, 236402 (2012).

${ }^{55}$ N. B. Slater, Nature (London) 180, 1352 (1957).

${ }^{56}$ V. F. Sears and S. A. Shelley, Acta Crystallogr., Sect. A 47, 441 (1991).

${ }^{57}$ H. E. Swanson, M. C. Morris, and E. Evans, Natl. Bur. Stand. Monogr. 25(4), 3 (1966).

${ }^{58}$ R. Kirby, T. A. Hahn, and B. D. Rothrock, American Institute of Physics Handbook, 3rd ed., edited by D. E. Gray (McGrw-Hill, New York, 1963).

${ }^{59}$ A. D. McNaught and A. Wilkinson, IUPAC: Compendium of Chemical Terminology, 2nd ed. (Blackwell Scientific Publications, Oxford, 1997). 